\title{
Effective apsidal precession in oblate coordinates
}

\author{
Abraão J. S. Capistrano ${ }^{1,2 \star}$, Paola T. Z. Seidel ${ }^{1} \dagger$, Luís A. Cabral $^{1,3} \ddagger$ \\ ${ }^{1}$ Applied physics graduation program, UNILA, 85867-670, P.o.b: 2123, Foz do Iguaçu-PR, Brazil \\ ${ }^{2}$ Casimiro Montenegro Filho Astronomy Center, Itaipu Technological Park, 85867-900, Foz do Iguaçu-PR, Brazil \\ ${ }^{3}$ Universidade Federal do Tocantins, Curso de Física, Setor Cimba, Araguaína - TO, 77824-838, Brazil
}

Accepted Received ; in original form

\begin{abstract}
We use oblate coordinates to study its resulting orbit equations. Their related solutions of Einstein's vacuum equations can be written as a linear combination of Legendre polynomials of positive definite integers $l$. Starting from solutions of the zeroth order $l=0$ in a nearly newtonian regime, we obtain a non-trivial formula favoring both retrograde and advanced solutions for the apsidal precession depending on parameters related to the metric coefficients, particularly applied to the apsidal precessions of Mercury and asteroids (Icarus and 2 Pallas). As a realization of the equivalence problem in general Relativity, a comparison is made with the resulting perihelion shift produced by Weyl cylindric coordinates and the Schwarzschild solution analyzing how different geometries of space-time influence on solutions in astrophysical phenomena.
\end{abstract}

Key words: perihelion, gravity

\section{INTRODUCTION}

Since the explanation of the perihelion advance of Mercury by Einstein in 1915 as application of general Relativity (GR), and it has been considered one of the fundamental laboratories for testing extensions of standard GR and other gravitational models such as, e.g, the modification of Newtonian Dynamics (MOND)(Schmidt 2008), Kaluza-Klein five-dimensional gravity (Lim and Wesson 1992), Yukawalike Modified Gravity (Iorio 2008a), Horava-Lifshitz gravity (Harko, 2011), brane-world models and variants (Mak and Harko 2004; Maia, Capistrano and Muller 2009; Cheung and Xu 2013; Chakraborty and Sengupta 2014; Jalalzadeh et al. 2009; Iorio 2009a,b) and in the parametric post-Newtonian $(\mathrm{PPN})$ framework and beyond and approaches in the weak field/slow motion limits (Avalos-Vargas and Ares de Parga 2012; Arakida 2013; Adkins and McDonnell 2007; Biswas and Mani 2005; D'Eliseo 2012; Deng and Xie 2014; Feldman 2013; Li et al. 2014; Iorio 2005, 2006, 2008b, 2011; Ruggiero 2014; Wilhelm and Dwivedi 2014).

This paper aims at showing the comparison of different geometries and on how it inflicts on the underlying physics to describe the same astrophysical phenomenon. This has a particular relevance for astrophysical phenomena where the form of the objects plays a central role to obtain a more realistic description and a departure from a spherical geometry may give more insight on the physical phenomena.

\footnotetext{
* E-mail:abraao.capistrano@unila.edu.br

$\dagger$ E-mail:paola.seidel@gmail.com

$\ddagger$ E-mail:cabral@uft.edu.br
}

This solution has to do with the shape, the topology or the symmetry aspects of the gravitational field. To do so, the arbitrary (diffeomorphic) transformations of GR cannot happen. This is a fine example of the equivalence problem in GR on how to know that solutions of Einstein's equations in different coordinates do not describe the same gravitational field. The application of Cartan's equivalence method (Cartan, 1927) solves this situation based on the fact that the Riemann tensors and their covariant derivatives up to the tenth order must be equal. However, another method also solves that issue using covariant derivatives up to the seventh order (Karlhede 1980). In the second section, we make a brief review of Zipoy's work on oblate static metric and the "monopole" solution that resides on the zeroth degree of Legendre polynomials. Moreover, an orbit equation is obtained. In the third section, the calculations of a nonstandard expression for the perihelion shift are shown with a comparison with the standard Einstein result and Weyl's axial metric. We also apply the model to asteroid in inner (Icarus) and outer solar system (2 Pallas). Finally, we make the final remarks in the conclusion section.

\section{ZIPOY OBLATE METRIC}

\subsection{Form and general solution of Zipoy's metric}

An interesting work published by Zipoy (1966) investigates some topological properties on oblate spheroidal and prolate coordinates by calculating the vacuum Einstein's equations to study general properties of the metrics such as their asymptotic behaviour, singularities and stability. Moreover, 


\section{Abraão J. S. Capistrano, Paola T. Z. Seidel, Luís A. Cabral}

he found that those metrics present a nearly newtonian ${ }^{1}$ solution resulting a linear combination of Legendre polynomials. Bearing in mind that the astrophysical phenomena depend on the form of objects, then different metrics must provide different aspects of the inner physics of the phenomena, once the diffeomorphic group of general relativity is broken, and diffeomorphic transformations cannot be allowed and new prospects may be found particularly on applications in astrophysics.

On the mechanism we are going to show, we consider the effects in a single plane of orbit. This consideration is compatible with the observed movement of the planets around the Sun limited to the plane of orbits. Considering the Sun in the center of the circular base of a cylinder and a planet (or a small celestial object) as a particle with mass $m$ orbiting its edge, it can be described by Weyl's line element (Weyl, 1917)

$d s^{2}=-e^{2(\lambda-\sigma)}\left(d \rho^{2}+d z^{2}\right)-\rho^{2} e^{-2 \sigma} d \phi^{2}+e^{2 \sigma} d t^{2}$,

where the coefficients $\lambda=\lambda(\rho, z)$ and $\sigma=\sigma(\rho, z)$ are the Weyl potentials. Moreover, this metric is diffeomorphic to the Schwarzschild's one, and it does not lose its asymptotes and is asymptotically flat (Weyl, 1917; Rosen, 1949; Zipoy 1966; Gautreau, Hoffman and Armenti, 1972; Stephani et al., 2003). Differently from the works of (González, Gutiérrez-Piñeres and Ospina, 2008; GutiérrezPiñeres, González and Quevedo, 2013; Ujevic and Letelier, 2004, 2007) and (Vogt and Letelier, 2008) where the authors use a mass distribution with Weyl's exact of Einstein equations, we studied approximate solutions of this metric for a test particle by expanding the metric coefficient functions (or potentials) into a Taylor's series and as a result the obtained perihelion shift was about 43.105 arcsec/century (Capistrano, Roque and Valada, 2014).

To obtain the oblate coordinates, a change of variable can be applied in such a form $\rho=a \cosh v \cos \theta$ and $z=$ $a \sinh v \sin \theta$, and $a$ is a length parameter. The resulting line element is given by

$$
\begin{array}{r}
d s^{2}=-a^{2} e^{2(\lambda-\sigma)}\left(\sinh ^{2} v+\sin ^{2} \theta\right)\left(d v^{2}+d \theta^{2}\right) \\
-a^{2} e^{-2 \sigma} \cosh ^{2} v \cos ^{2} \theta d \phi^{2}+e^{2 \sigma} d t^{2}
\end{array}
$$

where $(v, \theta)$ are the oblate coordinates, being the variation of $v$ producing ellipsoids intertwined by hyperboloids built by the coordinate $\theta$. In this case, the situation is physically more interesting, for instance, we can consider the Sun in one focus of the elliptical base in the plane of the orbit and the planets moving in this plane. Moreover, the exterior gravitational field in the cylinder outskirts is given by Einstein's vacuum equations

$$
\sigma_{, v v}+\sigma_{, \theta \theta}+\sigma_{, v} \tanh v-\sigma_{, \theta} \tan \theta=0
$$

1 We use the term nearly newtonian in the sense of Misner, Thorne and Wheeler (1973), and Infeld and Plebanski (1960), as an intermediate gravitational field between the general Relativity and Newtonian gravitational field in such a way that there is no a priori constraints on the field strength but only on the related movement (geodesic) equations. Needless to say, whenever the presuppositions of the weak field regime and the slow motion condition are applied and the expansion parameters of the metric are set, it leads naturally to the post-newtonian regime (Capistrano, 2018).

$$
\begin{aligned}
& \sigma_{, v}^{2}-\sigma_{, \theta}^{2}-\lambda_{, v} \tanh v-\lambda_{, \theta} \tan \theta=0, \\
& 2 \sigma_{, v} \sigma_{, \theta}+\lambda_{, v} \tan \theta-\lambda_{, \theta} \tanh v=0, \\
& \lambda_{, v v}+\lambda_{, \theta \theta}+\sigma_{, v}^{2}+\sigma_{, \theta}^{2}=0 .
\end{aligned}
$$

where the notation $(, v),(, \theta)$ and $(, v v),(, \theta \theta)$ denote respectively the first and the second derivatives with respect to the variables $v$ and $\theta$. Noting that eq.(3) is just Laplace's equation in oblate coordinates, a solution of the coefficient $\sigma$ can be found. Firstly, a change of variables can be made with $x=\sinh v$ and $y=\sin \theta$, and after using the method of separation of variables, one can write $\sigma(x, y)=P(x) Q(y)$, and find

$\frac{\partial}{\partial x}\left[\left(x^{2}+1\right) \frac{\partial \sigma}{\partial x}\right]+\frac{\partial}{\partial y}\left[\left(1-y^{2}\right) \frac{\partial \sigma}{\partial y}\right]$,

and their resulting separated equations

$$
\begin{aligned}
& \frac{\partial}{\partial x}\left[\left(x^{2}+1\right) \frac{\partial P(x)}{\partial x}\right]-l(l+1) P(x)=0, \\
& \frac{\partial}{\partial y}\left[\left(1-y^{2}\right) \frac{\partial Q(y)}{\partial y}\right]+l(l+1) Q(y)=0,
\end{aligned}
$$

where $l$ are the degree of Legendre polynomials. The solutions $P(x)$ and $Q(y)$ are given by the Legendre polynomials of first kind and both Legendre polynomials of first and (the complex) second kind, respectively. Due to the structure of the line element eq.(2), we only need the coefficient $\sigma$ to produce a nearly newtonian gravitational regime by the component $g_{44}$ (Misner, Thorne and Wheeler 1973). For this reason, we are only interested in the solution for the coefficient $\sigma$. Following the results in Zipoy (1966), for the "monopole" solution $l=0$, one can obtain:

$e^{2 \nu}=\left(\frac{r^{2}+a^{2} \sin \theta^{2}}{r^{2}+a^{2}}\right)^{\beta^{2}+1}$,

and the $\sigma(r)$ potential is given by

$\sigma(r)=-\beta \arctan \frac{a}{r}$,

being $0 \leqslant \arctan \frac{a}{r} \leqslant \pi, \beta=\frac{m}{a}$ and $r=a \sinh v$. The quantities $a$ and $m$ are length parameters, being $\beta$ a dimensionless quantity. Hereon, we consider only $a$ and $\beta$ as fundamental parameters for our further analysis. This new change of variable leads to the line element

$$
\begin{aligned}
d s^{2}= & -e^{2(\nu-\sigma)} d r^{2}-e^{2(\nu-\sigma)}\left(r^{2}+a^{2}\right) d \theta^{2} \\
& -e^{-2 \sigma}\left(r^{2}+a^{2}\right) \cos ^{2} \theta d \phi^{2}+e^{2 \sigma} d t^{2} .
\end{aligned}
$$

As a realization of the diffeomorphism invariance, Zipoy showed when $r \rightarrow \infty$, the equation (11) turns into an isotropic Schwarzschild line element and the set of coordinates $(r, \theta, \phi)$ turns the usual spherical coordinates.

\subsection{Orbit equation for the "monopole" solution $l=0$}

To start with, we consider a constraint to restrain the movement of a particle to the plane of the orbit setting the coordinate $\theta=0$ that imposes a constraint on the diffeomorphism invariance. Hence, we have a constraint on velocities

$\vec{v} \cdot \vec{v}=g_{\alpha \beta} v^{\alpha} v^{\beta}=-1$,

where we denote $v^{\alpha}=\frac{d \alpha}{d \tau}$. Thus, we also denote the quantities $v^{r}=\frac{d r}{d \tau}, v^{\phi}=\frac{d \phi}{d \tau}$, and $v^{t}=\frac{d t}{d \tau}$. Moreover, using eq. (11) 
and (12), one can obtain the following expression

$$
\begin{gathered}
-\left(\frac{r^{2}}{r^{2}+a^{2}}\right)^{\beta^{2}+1} e^{-2 \sigma(r)}\left(\frac{d r}{d \tau}\right)^{2} \\
-e^{-2 \sigma(r)}\left(r^{2}+a^{2}\right)\left(\frac{d \phi}{d \tau}\right)^{2}+e^{2 \sigma(r)}\left(\frac{d t}{d \tau}\right)^{2}=-1 .
\end{gathered}
$$

To proceed further, we need to know the conserved quantities. This can be obtained using the functional $\mathcal{L}=$ $\frac{1}{2} g_{\mu \nu} \dot{x}^{\mu} \dot{x}^{\nu}$ and the Euler-Lagrange equations,

$\frac{\partial \mathcal{L}}{\partial x^{\mu}}-\frac{d}{d \tau}\left(\frac{\partial \mathcal{L}}{\partial \dot{x}^{\mu}}\right)=0$

and for the interested case, we set the dependence of $\dot{x}^{\mu}$ for the coordinates $\phi$ and $t$. Hence, one finds

$$
\left(\frac{d \phi}{d \tau}\right)^{2}=\frac{L^{2} e^{4 \sigma(r)}}{\left(r^{2}+a^{2}\right)^{2}},
$$

and also

$$
\left(\frac{d t}{d \tau}\right)^{2}=E^{2} e^{-4 \sigma(r)}
$$

where we denote the conserved quantities $L$ for the specific orbital angular momentum and $E$ for the specific orbital energy. With those previous results, we can rewrite eq.(13) in a form

$$
\begin{array}{r}
-\left(\frac{r^{2}}{r^{2}+a^{2}}\right)^{\beta^{2}+1} e^{-2 \sigma(r)}\left(\frac{d r}{d \tau}\right)^{2}-\left(\frac{L^{2} e^{2 \sigma(r)}}{r^{2}+a^{2}}\right) \\
+e^{-2 \sigma(r)} E^{2}=-1,
\end{array}
$$

and after a little algebra, one finds

$$
\begin{gathered}
\left(\frac{d r}{d \phi}\right)^{2}=\left[1-\frac{L^{2} e^{2 \sigma(r)}}{\left(r^{2}+a^{2}\right)}+e^{-2 \sigma(r)} E^{2}\right] \\
\frac{e^{-2 \sigma(r)}}{L^{2}}\left(\frac{r^{2}+a^{2}}{r^{2}}\right)^{\beta^{2}+1}\left(r^{2}+a^{2}\right)^{2} .
\end{gathered}
$$

Taking a change of variable $u=\frac{1}{r}$, we can find an orbit equation

$$
\begin{gathered}
\left(\frac{d u}{d \phi}\right)^{2}=-u^{2}\left(1+a^{2} u^{2}\right)^{\beta^{2}+2} \\
+\frac{e^{-2 \sigma(u)}}{L^{2}}\left(1+a^{2} u^{2}\right)^{\beta^{2}+3}\left[1+E^{2} e^{-2 \sigma(u)}\right] .
\end{gathered}
$$

and developing the previous equation, we have

$$
\begin{array}{r}
\left(\frac{d u}{d \phi}\right)^{2}=-u^{2}\left(1+2 a^{2} u^{2}+a^{4} u^{4}\right)\left(1+a^{2} u^{2}\right)^{\beta^{2}} \\
+\frac{e^{-2 \sigma(u)} C(u)}{L^{2}}\left(1+a^{2} u^{2}+2 a^{2} u^{2}+2 a^{4} u^{4}\right)\left(1+a^{2} u^{2}\right)^{\beta^{2}} \\
+\frac{e^{-2 \sigma(u)} C(u)}{L^{2}}\left(a^{4} u^{4}+a^{6} u^{6}\right)\left(1+a^{2} u^{2}\right)^{\beta^{2}}
\end{array}
$$

where we denote $C(u)=1+E^{2} e^{-2 \sigma(u)}$. Equivalently, we can write

$$
\begin{array}{r}
\left(\frac{d u}{d \phi}\right)^{2}=\alpha(u) u^{2}\left[\frac{3 a^{2} C(u)}{e^{2 \sigma(u)} L^{2}}-1\right] \\
+\alpha(u) a^{2} u^{4}\left[\frac{3 a^{2} C(u)}{e^{2 \sigma(u)} L^{2}}-2\right]+\alpha(u) a^{4} u^{6}\left[\frac{a^{2} C(u)}{e^{2 \sigma(u)} L^{2}}-1\right] \\
+\frac{\alpha(u) C(u)}{e^{2 \sigma(u)} L^{2}}
\end{array}
$$

where we denote $\alpha(u)=\left(1+a^{2} u^{2}\right)^{\beta^{2}}$. Hence, a more convenient form for the resulting orbit equation can be written as

$$
\begin{array}{r}
\left(\frac{d u}{d \phi}\right)^{2}+u^{2}=\alpha(u) u^{2}\left[\frac{3 a^{2} C(u)}{e^{2 \sigma(u)} L^{2}}-1\right] \\
+\alpha(u) a^{2} u^{4}\left[\frac{3 a^{2} C(u)}{e^{2 \sigma(u)} L^{2}}-2\right]+\alpha(u) a^{4} u^{6}\left[\frac{a^{2} C(u)}{e^{2 \sigma(u)} L^{2}}-1\right] \\
+\frac{\alpha(u) C(u)}{e^{2 \sigma(u)} L^{2}}+u^{2} .
\end{array}
$$

It is noteworthy to point out that this equation is a highly non linear type, even in the simplest "monopole" case with $l=0$ and $\theta=0$.

\section{ANALYSIS ON APSIDAL PRECESSION}

To work with eq.(22), we attenuate the field strength by analyzing the decaying terms and by the magnitude of the $\beta$ parameter, which is related to the coefficient $\sigma$ by eq.(10). Firstly, we start truncating high orders of the variable $u$ constrained to $u^{4}$, since the effects $\mathcal{O}\left(u^{5}\right)$ in solar system scale are negligible (Yamada and Asada, 2012). Hence,

$$
\begin{aligned}
\left(\frac{d u}{d \phi}\right)^{2}+u^{2}= & \alpha(u) u^{2}\left[\frac{3 a^{2} C(u)}{e^{2 \sigma(u)} L^{2}}-1\right]+\alpha(u) a^{2} u^{4} \\
& {\left[\frac{3 a^{2} C(u)}{e^{2 \sigma(u)} L^{2}}-2\right]+\frac{\alpha(u) C(u)}{e^{2 \sigma(u)} L^{2}}+u^{2} . }
\end{aligned}
$$

Due to the fact that the previous orbit equation still remains strongly nonlinear, we can study approximate solutions if we impose that the parameter $\beta$ is small, then the length parameter $a$ must be large. Moreover, for small values of the $\beta$ parameter, the term $\alpha(u)$ can be expanded as $\alpha(u)=$ $1+\beta^{2} a^{2} u^{2}+O(u)^{3}$. We point out that for orders of $u^{3}$ and on, it will produce terms of orders higher than $u^{4}$ in the main equation in eq.(23), so the expansion in the term $\alpha(u)$ is limited to $u^{2}$. On the other hand, since $E$ should be the specific orbital energy, from the term $C(u)$ we find that $E^{2} e^{-2 \sigma(u)}>>1$. These two considerations lead us to a more treatable orbit equation in such a form

$$
\begin{aligned}
\left(\frac{d u}{d \phi}\right)^{2}+u^{2} & =u^{2}\left[\frac{3 a^{2} E^{2}}{e^{4 \sigma(u)} L^{2}}-1\right]+u^{4} a^{2} \beta^{2}\left[\frac{3 a^{2} E^{2}}{e^{4 \sigma(u)} L^{2}}-1\right] \\
& +a^{2} u^{4}\left[\frac{3 a^{2} E^{2}}{e^{4 \sigma(u)} L^{2}}-2\right]+\frac{\left(1+a^{2} u^{2} \beta^{2}\right) E^{2}}{e^{4 \sigma(u)} L^{2}}+u^{2}
\end{aligned}
$$

With the fact that the variable $u$ can be related with the oblate angles in such a way $r=a x=a \sinh v$, from eq.(10), we can write $e^{-4 \sigma(v)}=e^{4 \beta \arctan (\operatorname{csch} v)}$. This allows us to study a closed positive infinite endpoints of the orbit where $v=[0,+\infty]$. At $v \rightarrow+\infty$, the ellipsoid approaches to a circular orbit and at $v \rightarrow 0$ it approaches to a ring singularity (Zipoy 1966), as illustrated in fig.(1). Then elliptic trajectories can be studied in-between from their respective endpoints, since the potential $\sigma$ does remain finite. Hence, using eq.(10) and examining the tendencies, close to circular orbits with $v \rightarrow+\infty$, then $\sigma(u)$ approaches 0 , and the exponential term $e^{-4 \sigma(v)}$ approaches 1 . On the other hand, close to singularity, one can expand the related functions around zero $(v \rightarrow 0)$ of the argument of the exponential that leads to $-4 \sigma(v)=-2 \beta \operatorname{sgn}(1 / v) \pi-v=-2 \beta \operatorname{sgn}(+\infty) \pi=-2 \beta \pi$, and the exponential term approaches $e^{-2 \beta \pi}$, where $\operatorname{sgn}$ is 

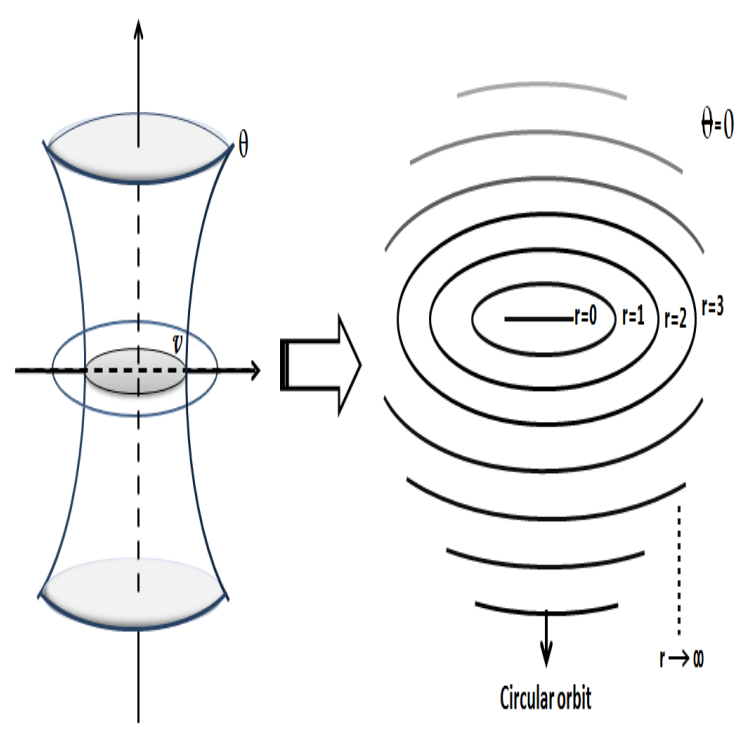

Figure 1. Pictorial view of the oblate coordinates int the plane $(v, \theta)$ with a hyperboloid and centered ellipsoid. In the right figure, it is shown a reduction of the oblate coordinates into a two dimensional plane with $\theta=0$. In this case, we have a two dimensional ellipsoid where $r \rightarrow 0$ is transformed into a singular ring (in the sense of Riemann invariants are infinite). In the case $r \rightarrow \infty$, the elliptical plane approaches to a circular plane.

the sign function. Thus, one can obtain two orbit equations in such a limits, respectively,

$$
\begin{gathered}
\left(\frac{d u}{d \phi}\right)^{2}+u^{2}=\frac{E^{2}}{L^{2}}+u^{2} \frac{a^{2} E^{2}}{L^{2}}\left(3+\beta^{2}\right) \\
+u^{4} a^{2}\left(\beta^{2}\left[\frac{3 a^{2} E^{2}}{L^{2}}-1\right]+\left[\frac{3 a^{2} E^{2}}{L^{2}}-2\right]\right), \\
\left(\frac{d u}{d \phi}\right)^{2}+u^{2}=\frac{E^{2}}{L^{2}} e^{-2 \beta \pi}+u^{2} \frac{a^{2} E^{2}}{L^{2}} e^{-2 \beta \pi}\left(3+\beta^{2}\right) \\
+u^{4} a^{2}\left(\beta^{2}\left[\frac{3 a^{2} E^{2}}{L^{2}} e^{-2 \beta \pi}-1\right]+\left[\frac{3 a^{2} E^{2}}{L^{2}} e^{-2 \beta \pi}-2\right]\right) .
\end{gathered}
$$

Using the method as shown in (Harko, 2011), we can work with the previous orbit equations analytically. In the first case with $v \rightarrow+\infty$ close to circular orbits, we can write

$\left(\frac{d u}{d \phi}\right)^{2}+u^{2}=u^{2} A+u^{4} B+D=G(u)$,

where $A, B$ and $D$ are respectively

$A=\frac{a^{2} E^{2}}{L^{2}}\left(3+\beta^{2}\right)$,

$B=a^{2} \beta^{2}\left[\frac{3 a^{2} E^{2}}{L^{2}}-1\right]+a^{2}\left[\frac{3 a^{2} E^{2}}{L^{2}}-2\right]$

$D=\frac{E^{2}}{L^{2}}$,

where the deviation angle $\delta \phi$ can be found using

$\delta \phi=\left.\pi \frac{d F(u)}{d u}\right|_{u_{0}}$,

with the constraint $F\left(u_{0}\right)=u_{0}$ and $F(u)$ is denoted by
$F(u)=\frac{1}{2} \frac{d G(u)}{d u}$.

With those informations at hand, we can evaluate $F(u)$ straightforwardly

$F(u)=\frac{1}{2} \frac{d G(u)}{d u}=\frac{1}{2}\left(2 u A+4 u^{3} B\right)=u A+2 u^{3} B$,

and the related algebraic equation

$u_{0} A+2 u_{0}^{3} B=u_{0}$,

with solution

$u_{0}=\sqrt{\frac{1-A}{2 B}}$.

By using eq.(30), we find a deviation from a closed circular orbit as

$\delta \phi=-2 \pi\left(\frac{a^{2} E^{2}}{L^{2}}\left(3+\beta^{2}\right)\right)$.

Likewise, for the second case, close to singularity $(v \rightarrow 0)$, we can find the deviation angle $\delta \phi *$ by the orbit equation in a form

$\left(\frac{d u}{d \phi}\right)^{2}+u^{2}=u^{2} H+u^{4} J+N$

with $H, J$ and $N$ respectively

$H=\frac{a^{2} E^{2}}{L^{2}}\left(3+\beta^{2}\right) e^{-2 \beta \pi}$,

$J=a^{2} \beta^{2}\left[\frac{3 a^{2} E^{2}}{L^{2}} e^{-2 \beta \pi}-1\right]+a^{2}\left[\frac{3 a^{2} E^{2}}{L^{2}} e^{-2 \beta \pi}-2\right]$,

and

$N=\frac{E^{2}}{L^{2}} e^{-2 \beta \pi}$.

Conversely,

$F(u)=\frac{1}{2} \frac{d G(u)}{d u}=\frac{1}{2}\left(2 u H+4 u^{3} J\right)=u H+2 u^{3} J$,

and the associated algebraic equation

$u_{0} H+2 u_{0}^{3} J=u_{0}$,

with a similar solution

$u_{0}=\sqrt{\frac{1-H}{2 J}}$,

implies the resulting deviation angle $\delta \phi *$ from a closed circular orbit that is given by

$\delta \phi *=-2 \pi\left(\frac{a^{2} E^{2}}{L^{2}}\left(3+\beta^{2}\right)\right) e^{-2 \beta \pi}$.

A good estimate of the effective deviation angle can be obtained by the asymptotic matched expansions between eq.(42) and eq.(35) given by

$$
\delta \phi_{\text {eff }}=\delta \phi+\delta \phi *-\delta \phi_{\text {overlapped }},
$$

where $\delta \phi_{\text {overlapped }}$ denotes the resulting angle when the solutions $\delta \phi$ and $\delta \phi *$ are overlapped, and it occurs when $\beta=0$. As a result, it lead us to the "Zipoy's precession formula" given by the deviation angle in the elliptical plane of the orbits

$\delta \phi_{(z i p)}=-2 \pi \frac{a^{2} E^{2}}{L^{2}}\left(3 e^{-2 \beta \pi}+\beta^{2}\left(1+e^{-2 \beta \pi}\right)\right)$. 
Table 1. Comparison between the values for secular precession of Mercury in units of arcsec/century $\left({ }^{\prime \prime} . c y^{-1}\right)$ of the standard (Einstein) perihelion precession $\delta \phi_{s c h}$ (Wilhelm and Dwivedi 2014) and the Weyl conformastatic solution $\delta \phi_{W e y l}$. The $\delta \phi_{o b s}$ stands for the secular observed perihelion precession in units of arcsec/century. In the fourth column, some observational values of perihelion precession are available. The first data point was adapted from (Nambuya 2010) by adding a supplementary precession calibrated with the Ephemerides of the Planets and the Moon (EPM2011) (Pitjeva and Pitjev 2013; Pitjev and Pitjeva 2013).

\begin{tabular}{|c|c|c|c|c|}
\hline$\delta \phi_{\text {sch }}$ & $\delta \phi_{W e y l}$ & $\delta \phi_{Z i p o y}$ & $\delta \phi_{o b s}$ & References \\
\hline 42.9781 & 43.105 & 42.9696 & $\begin{aligned} 43.098 & \pm 0.503 \\
43.20 & \pm 0.86 \\
43.11 & \pm 0.22 \\
43.11 & \pm 0.22 \\
42.98 & \pm 0.09 \\
43.13 & \pm 0.14 \\
42.98 & \pm 0.04 \\
43.03 & \pm 0.00 \\
43.11 & \pm 0.45\end{aligned}$ & $\begin{array}{l}\text { (Nambuya 2010; Pitjeva and Pitjev 2013; Pitjev and Pitjeva 2013) } \\
\text { (Shapiro et al., 1972) } \\
\text { (Shapiro, Counselmann III and King, 1976) } \\
\text { (Anderson et al. 1978) } \\
\text { (Shapiro et al., 1990) } \\
\quad \text { (Anderson et al. 1991) } \\
\text { (Nobili and Will 1986; Will, 2006) } \\
\quad \text { (Clemence, 1964) } \\
\text { (Duncombe 1956; Morton 1956) }\end{array}$ \\
\hline
\end{tabular}

Table 2. Comparison between the observational values $\delta \phi_{o b s}$ for secular precession in units of arcsec/century and the values from the standard (Einstein) perihelion precession and the Zipoy solution $\delta \phi_{\text {model }}$ for selected 1566 Icarus asteroid and 2 Pallas.

\begin{tabular}{llrr}
\hline Object & $\delta \phi_{\text {obs }}\left({ }^{\prime \prime} . c y^{-1}\right)$ & $\delta \phi_{\text {sch }}\left({ }^{\prime \prime} . c y^{-1}\right)$ & $\delta \phi_{\text {model }}\left({ }^{\prime \prime} . c y^{-1}\right)$ \\
\hline 1566 Icarus & 10.05 & 10.0613 & 10.029 \\
2 Pallas & -133.534 & - & -133.52 \\
\end{tabular}

Interestingly, the solution provides a retrograde precession besides the advanced one and the result is set by the conserved quantities and parameters initially considered. It is noteworthy to point out that the hyperbolic term persists in the result evincing the propagation of the non linear effects from the Einstein equations even with the breakage of the diffeomorphic coordinate transformations.

To obtain the correct physical units, we use the known forms for the specific orbital energy $E=\frac{-G M}{2 \gamma}$ and the specific orbital momentum $L^{2}=\mu p$, with $\mu=G M$ and $p=\gamma\left(1-\epsilon^{2}\right)$. The terms $M, \gamma$ and $\epsilon$ denote the central Sun mass, the semi-major axis and the orbital eccentricity, respectively. The Newton's universal gravitational constant is denoted by $G$. Since $\beta$ is small, the hyperbolic exponential can be approximated to $e^{-2 \beta \pi} \sim 1-2 \beta \pi$. It is important to stress that high orders on $\beta$ are neglected. Accordingly, using eq.(43), one can obtain

$\delta \phi_{(z i p)}=-\frac{3}{2} \pi \frac{a^{2} G M}{c^{2} \gamma^{3}\left(1-\epsilon^{2}\right)}(1-2 \beta \pi)$.

A more familiar expression for apsidal precession can be obtained by using the orbital period $P$ in days in such a way we have the final form

$\delta \phi_{(z i p)}=\frac{-6 \pi^{3} a^{2}}{c^{2}\left(1-\epsilon^{2}\right) P^{2}}(1-2 \beta \pi)$,

which resembles the standard Schwarzschild formula. For the physical quantities, we adopt the international system of measurement Bureau International des Poids et Mesures (Bureau International des Poids et Mesures 2006) setting one year $1 y r=365.256 d$, the speed of light $c=$ $299792458 \mathrm{~m} / \mathrm{s}$ (Wilhelm and Dwivedi 2014; Bureau Inter- national des Poids et Mesures 2006) and the mass of sun $M_{\odot}=1.98853 \times 10^{30} \mathrm{~kg}$. The period $P$ is given by $P=$ $T(24)(3600)$ and $T$ is the sidereal orbital period in days. In the case of Mercury, we use $T=87.969$ days (NASA Mercury Fact Sheet. https://nssdc.gsfc.nasa.gov).

We use 9 data points concerning observations on the perihelion advance of Mercury in units of arcsecond per century $\left({ }^{\prime \prime} . c y^{-1}\right)$ as shown in table 01 . We denote $\delta \phi_{s c h}$ for standard (Einstein) perihelion precession and $\delta \phi_{W e y l}$ for the resulting perihelion advance using the Weyl conformastatic solution (Capistrano, Roque and Valada, 2014), which comes from an axially-symmetric motion of a test particle in Weyl's line element (Weyl, 1917). To control the systematics, we use GnuPlot 5.2 software to compute non-linear least-squared fitting by using the Levenberg-Marquardt algorithm for the goodness-of-fitting to data. The obtained values for the parameters and the related reduced chi-squared $\left(\chi_{\text {red }}^{2}\right)$. Since eq.(45) has a negative sign, and to obtain an advanced precession solution, we calculate its absolute value. We observed that running the parameters without any priors, we find that the $a$ parameter has the same magnitude of the planetary semi-major axis as it provides $a \sim-1.15806 \times 10^{11}$, which its absolute value is roughly close to observational value of Mercury's semi-major axis and $\beta=8.86038 \times 10^{-6}$ and the resulting value for the shift angle is $42.9696^{\prime \prime} . c y^{-1}$ for a $\chi_{\text {red }}^{2}=0.0166$ and a probability $p>0.95$, which represents a good fitting. It is worth noting that the negative sign for the length parameter $a$ is a relic from the hyperbolic geometry that passed through the non linear effects from the initially strong gravitational field.

In table (1), the secular precession of Mercury in units of arcsec/century, comparing with standard Schwarzschild and 


\section{Abraão J. S. Capistrano, Paola T. Z. Seidel, Luís A. Cabral}

cylindric Weyl solutions for the perihelion shift, the obtained perihelion shift $\delta \phi_{\text {Zipoy }}$ reproduces closely the observed perihelion shift with a bonus that it naturally provides elliptical orbits which makes this solution a better physical description for astrophysical purposes according to the shape, the topology and the symmetry aspects of the gravitational field.

Departing from a spherical geometry, we are able to study precession of two asteroids. The first one corresponds to the Icarus asteroid. This asteroid is a near-Earth object (NEO) of the Apollo group with a very elliptical orbit. It has been regarded as a relativistic asteroid with an approximation even close to the Sun than Mercury and also a Venus and Mars-crosser. Its observational value for the perihelion precession is 10.05 arcseconds per century with semi-major axis $1.61258 \times 10^{11} \mathrm{~m}$ and a large eccentricity 0.82695 for an orbital period $T=408.781$ days (Wilhelm and Dwivedi 2014). As a result, we obtained the values for the parameters $a \sim-3.21987 \times 10^{11}$ and $\beta=8.0222 \times 10^{-6}$ that provide a value for the shift angle $10.029^{\prime \prime} . c y^{-1}$ for $\chi_{\text {red }}^{2}=0.00272$ and $p>0.95$.

In addition, as an example of retrograde precession, which is not accounted for Einstein standard perihelion formula, we studied the 2 Pallas protoplanet, even though the available information on 2 Pallas are still scarce. The 2 Pallas asteroid is one of the largest asteroids in asteroid belt and is a Jupiter-crosser. Its observational value for the perihelion precession is -1333.534 arcseconds per century with semi-major axis $4.14520 \times 10^{11} \mathrm{~m}$ and a large eccentricity 0.2812 for an orbital period $T=1686.43$ days (available at http://hamilton.dm.unipi.it/astdys/index.php?pc=0, Asteroids Dynamic Site- AstDyS ). As a result, we obtained the values for the parameters $a \sim-1.680 \times 10^{13}$ and $\beta=8.0222 \times 10^{-6}$ that provide a value for the shift angle $-133.481^{\prime \prime} . c y^{-1}$ for $\chi_{\text {red }}^{2}=1245.46$ and $p>0.95$. In the two previous cases, the value of the $\beta$ parameter remains the same and unless we find a counterproof, its value around $\sim 10^{-6}$ must remain the same for any large object in Solar system.

It reinforces the main aspect of this paper on "equivalence problem" in GR. As commented previously, the Weyl and Zipoy metric are asymptotically convergent to Schwarzschild coordinates but once the diffeomorphic invariance is not allowed, the produced gravitational fields are not the same, and they can be adjusted to a specific ending. In this case, the Zipoy seems to be a more physically appropriate solution as compared to the standard Einstein or PPN solution.

\section{FINAL REMARKS}

Our results in this paper is a fine example that the nonlinearities of a system of equations imprint qualitative effects on the orbits of their solutions. We have studied solutions of vacuum Einstein's equation of an oblate metric obtaining a set of solutions that depends on the Legendre Polynomials, as shown by Zipoy in his seminal paper (Zipoy 1966). In hindsight, the simplest studied solution was the so-called "monopole" solution for the zeroth order of Legendre polynomials $l=0$. Starting from the related Lagrange equations, we have obtained the orbit equations, which revealed to be a highly non linear equation. To obtain an analytical solution, we have studied a closed positive infinite interval to obtain the elliptical pattern of the orbits in-between. As a result, we have obtained a non-standard expression for the perihelion precession depending on the dimensionless parameter $\beta$ and the length parameter $a$. The $\beta$ parameter was primarily fixed as a low magnitude to allow us to study the orbit equation. It is worth noting to point out that no a priori assumptions concerning the strength of the field (as a weak field) were imposed. Moreover, the values of the length parameter $a$ were adjust numerically using the Chi-square statistics for 9 observational data sets. We have shown the length parameter, as posed by Zipoy, can be attributed to it a physical meaning since it is close related to semi-major axis. Interestingly, the values converged to the same order of magnitude of semi-major axis of Mercury. Differently from the standard Einstein's solution and the Weyl cylindrical one, the precession formula from oblate coordinates provides naturally both retrograde and advanced solutions for the perihelion precession besides the fact that elliptical orbits are also native in those coordinates, which reinforces the idea that the topological nature of the problem is now an important character and the strength of the gravitational field is constrained by this topology. In summary, this analysis was made in the realm of RG in a nearly newtonian limit with no need of additional extensions or modifications of the standard gravity. As future perspectives, the extended analysis of the deviation of light, radar echo and gravitational lens in oblate and prolate metrics are currently in progress.

\section{ACKNOWLEDGMENTS}

Paola T.Z. Seidel thanks the Coordination for the Improvement of Higher education Personnel (Capes-Brazil) and the Fundação Araucária/PR for the scholarship grant Capes/FA (Chamada Pública 19/2015). We also thank Carlos Coimbra and Rodrigo Bloot for fruitful discussions and criticisms.

\section{REFERENCES}

Anderson J.D., Keesey M.S.W., Lau E.L., Standish E.M., Newhall X.X., 1978, Acta Astronautica, 5, 4361.

Anderson J.D., Campbell J.K., Jurgens R.F., Lau E.L., Newhall X.X., Slade III M.A., Standish Jr E.M., 1992, "Recent developments in solar-system tests of general relativity", In: H. Sato et al (Eds.): Proceedings of the 6th Marcel Grossmann Meeting on General Relativity, Kyoto, June 1991, World Scientific, Singapore, 353355.

Avalos-Vargas A., Ares de Parga, G., 2012, EPJ Plus, 127, 155.

Arakida H., 2013, Int. J. Theor. Phys., 52, 5, 1408-1414.

Adkins G. S.; McDonnell J., 2007, Phys. Rev. D75, 8, 082001.

Biswas A.; Mani K., 2005, Centr.Eur.Jour.Phys., 3(1), 6976 .

Bureau International des Poids et Mesures, 2006. Le Systéme International d'Unités(SI), 8e édition, BIPM, Sévres, p. 37.

Capistrano A.J.S., Roque W.L., Valada R.S., 2014, Mon. Not. R. Astron. Soc., 444, 1639-1646. 
Capistrano A.J.S., Penagos J.A.M, Alárcon M.S., 2016, Mon. Not. R. Astron. Soc., 463, 1587-1591.

Capistrano A.J.S., Galaxies 2018, 6, 48.

Cartan E., 1927, Ann. Soc. Pol. Mat 6, 1.

Clemence G.M., 1964, Icarus 3, 502.

Chakraborty S., Sengupta S., 2014, Phys. Rev. D 89, 026003.

Cheung Y.K.E., Xu F., 2013, Astrophys.J., 774, 1, 65, 20. D'Eliseo M. M, 2012, Astrophys. Space Sci., 342, 1, 15-18. Deng X., Xie Y., 2014, Astrophys. Space Sci., 350, 1, 103107.

Duncombe R.L., 1956, Astron. J. 61, 174175.

Feldman M.R., 2013, PLoS ONE, 8, 11, e78114.

Gautreau R., Hoffman R.B., Armenti A., 1972, Il Nuovo Cimento B, 7(1), 71-98.

Gergerly L.A. et al., 2011, Mon. Not. R. Astron. Soc., 415, 3275-3290.

González G.A., Gutiérrez-Piñeres A.C., Ospina P.A., 2008, Phys. Rev. D, 78, 064058.

Gutiérrez-Piñeres A.C., González G.A., Quevedo H., 2013, Phys. Rev. D, 87, 044010.

Harko T., 2011, Mon. Not. R. Astron. Soc., 413, 4, 30953104.

Infeld L., Plebanski J., 1960, Motion and Relativity, Pergamon Press.

Iorio L., 2008, Scholarly research Exchange Volume 2008, 238385 .

Iorio L., 2009, A.J., 137, 3615.

Iorio L., 2009, Int. J. Mod. Phys. D18, 947.

Iorio L., 2005, Class.Quant.Grav., 22, 24,5271-5281.

Iorio L., 2006, JCAP, 05, 002.

Iorio L., 2008, Advances in Astronomy, 268647.

Iorio L. et al., 2011, Astrophys. Space Sci., 331, 2, 351-395.

Jalalzadeh S. et al., 2009, Class.Quant.Grav., 26, 155007.

Karlhede A., 1980, Gen. Rel. Grav., 12, 693-707.

Li Z. et al., 2014, RAA, 14, 2, 139-143.

Lim P.H., Wesson P.S., 1992, Astrophys.J., 397, L91-L94.

Mak M.K., Harko T., 2004, Phys.Rev.D70, 024010.

Maia M.D.; Capistrano, A.J.S.; Muller D., 2009, IJMPD, 18, 1273-1289.

Misner C., Thorne K.S., Wheeler J.A., 1973, Gravitation, W.H. Freeeman \& Co.

Morton D.C., 1956, J.R. Astron. Soc. Canada 50, 223

Nambuya G.G., 2010, Mon. Not. R. Astron. Soc., 403, 1381.

Nobili A.M., Will C.M., 1986, Nature, 320, 39-41.

Pitjeva E. V., Pitjev N.P., 2013, Mon. Not. R. Astron. Soc., 432, 4, 3431-3437.

Pitjev N. P., Pitjeva, E. V., 2013, Astron. Lett., 39, 3, 141149.

Rosen N., 1949, Rev. Mod. Phys., 21, 503.

Ruggiero M. L., 2014, Int. J. Mod. Phys. D23, 5, 1450049.

Schmidt H.J., 2008, Phys. Rev.D 78, 023512.

Shapiro I.I., Pettengill G.H., Ash M.E., Ingalls R.P., Campbell D.B., Dyce R.B., 1972, Phys. Rev. Lett., 28, 15941597.

Shapiro I.I, Counselman III C.C., King R.W., 1976, Phys. Rev. Lett., 36, 555585.

Shapiro, I.I., 1990, "Solar system tests of general relativity: Recent results and present plan", In: N. Ashby et al (Eds.): General Relativity and Gravitation, 1989, Proceedings of the 12th International Conference on General
Relativity and Gravitation, Cambridge University Press, Cambridge, 313330.

Stephani H., Kramer D., MacCallum M., Hoenselaers C., Herlt E., 2003, Exact Solutions of Einstein's Field Equations. Cambridge: Cambridge University Press.

Wilhelm K., Dwivedi B. N., 2014, New Astronomy, 31, 5155.

Ujevic M., Letelier P.S., 2004, Phys. Rev. D, 70, 084015.

Ujevic M., Letelier P.S., 2007, Gen.Rel.Grav., 39, 13451365.

Vogt D., Letelier, P.S., 2008, Mon. Not. R. Astron. Soc., 384, 834-842.

Weyl H., 1917, Ann. Phys., 359, 117.

Will C.M., 2006, Living Rev. Relativity 9, 3, URL (accessed 29 May 2018).

Wilhelm K., Dwivedi B. N., 2014, New Astronomy, 31, 5155.

Zipoy M.D., 1966, Jour. Math. Phys., 7, 1137.

Yamada K., Asada H., 2012, Mon. Not. R. Astron. Soc., 423, 3540-3544. 